\title{
Network reconstruction in the presence of unmeasured neurons
} Duane Q Nykamp*

Address: Department of Mathematics, University of Minnesota, Minneapolis, MN 55455, USA

Email: Duane Q Nykamp* - nykamp@math.umn.edu

* Corresponding author

from Sixteenth Annual Computational Neuroscience Meeting: CNS*2007

Toronto, Canada. 7-12 July 2007

Published: 6 July 2007

BMC Neuroscience 2007, 8(Suppl 2):P20 doi:10.1 186/I47|-2202-8-S2-P20

(c) 2007 Nykamp; licensee BioMed Central Ltd.

We present a method to determine whether a correlation in the spikes of two neurons is due to a causal connection between the neurons or due to common input originating from unmeasured neurons. The distinction is based on a point-process model of how a neuron's spiking probability can depend on both its own spiking history and a stimulus (or other external variables). Although the results depend on selecting a parametric model that captures essential features of the neural response, a large class of models can be used with the network analysis. Hence, the analysis could be applied to probe circuitry in a large range of neuronal systems. 